\title{
Luminescence Properties of Manganese Doped CdS Nanoparticles under Various Synthesis Conditions
}

\author{
S. Salimian and S. Farjami Shayesteh* \\ Nanostructure Lab., Physics Department, University of Guilan, Rash, Iran \\ (Received August 1, 2009; revised version March 5, 2010; in final form May 3, 2010)
}

\begin{abstract}
An aqueous solution method has been developed for synthesizing size-controlled $\mathrm{Mn}^{2+}$ doped CdS nanocrystals with a relatively narrow size distribution. The nanocrystal samples were characterized by UV-Vis absorption spectra and photoluminescence spectra. We prepared narrow size distribution particles under different synthesis conditions. The effect of manganese concentration on the photoluminescence properties was investigated. Luminescence intensity in different excitation wavelength correlates with different size of CdS:Mn nanocrystals on luminescence spectra. We found that by narrowing the size distribution and doping concentration, CdS samples can be prepared with high luminescence intensity.
\end{abstract}

PACS numbers: 73.61.Ga, 74.25.Gz, 78.55.Et, 81.16.Be

\section{Introduction}

Nanoscale materials researches have stimulated great interest owing to their importance in basic scientific research and potential technological applications. The synthesis of doped nanocrystals (NCs) has become a major field of recent researches. Manganese doped CdS NCs have been mainly studied due to the luminescence of the $\mathrm{Mn}^{2+}$ ions inside the CdS host. This is due to the fact that $\mathrm{Mn}^{2+}$ ions provide good traps for the excited electrons, which give rise to their potential use in nonlinear optics, optoelectronic devices, solar cells, photodetectors and light-emitting diodes [1-7]. By doping NCs, the impurity states can play a special role in affecting the electronic energy structures and transient probabilities. In doped NCs the luminescence quantum efficiency is expected to increase as a result of a greater interaction between the electron and the hole of the host semiconductor material with the localized dopant levels [8]. However, effect of Mn doping on the structural phase and size control and optical properties of CdS nanostructures is still an issue and many groups have focused on this problems.

This paper presents some interesting results obtained on the synthesis of CdS:Mn nanocrystals by the aqueous solution method with controllable and narrow size distribution. We treat the effect of Mn doping on the luminescence properties of CdS nanoparticles. Also, we consider the effect of various synthesizing conditions on the narrow size distribution of the CdS particles and the luminescence intensity.

\section{Experimental}

Aqueous solutions of cadmium chloride, manganese chloride and the capping agent thioglycerol (TG) of high

* corresponding author; e-mail: saber@guilan.ac.ir
$(0.1 \mathrm{M})$ and low $(0.01 \mathrm{M})$ concentrations are prepared in ethanol. The $\mathrm{pH}$ was adjusted to about $2-10$ by adding appropriate amounts of $\mathrm{NaOH}$, before adding TG. Sodium sulfide dissolved in distilled water was added dropwise to the mixture for $5 \mathrm{~min}$. The total reaction time was about $2 \mathrm{~h}$. Nitrogen gas was used for deoxygenating the reaction vessel. The temperature in different experiments ranged between $27^{\circ} \mathrm{C}$ and $76^{\circ} \mathrm{C}$. In high temperature experiments, the synthesis vessel was heated in the bath. The synthesis solution was washed with acetone to get rid of unreacted ions remaining outside the clusters and then was centrifuged. Finally, the precipitate was air-dried to get a powder of CdS:Mn nanoparticles. The optical absorption spectra of CdS:Mn nanocrystallites dispersed in ethanol were recovered using a UV-Vis spectrophotometer (Cary 100). The luminescence spectra were recorded on a LF-5 Perkin Elmer spectrometer with the excitation wavelengths of about $370 \mathrm{~nm}$ to excite the CdS:Mn nanocrystals.

\section{Results and discussion}

For a typical nanoparticle sample, luminescence can be generally divided into band edge emission, including excitonic emission, and trap state emission. The luminescence spectra of the CdS:Mn nanocrystals are shown in Figs. 2-5. It was found that the luminescence spectra for all samples could be deconvoluted into two individual components, which corresponded to band edge emission and surface trap state emission [9]. As shown in Fig. 1, the mechanism of luminescence in CdS:Mn nanostructure can be explained as following: after excitation, energy will be transferred from the conduction band of CdS host to the excited charge carriers which are trapped in shallow trap states. These trapped charge carriers are followed by either energy transfer to the ${ }^{4} T_{1}$ excited state of a $\mathrm{Mn}^{2+}$ ion (Fig. 1), or radiative recombination with a deeply trapped hole at a defect state [10-14]. 


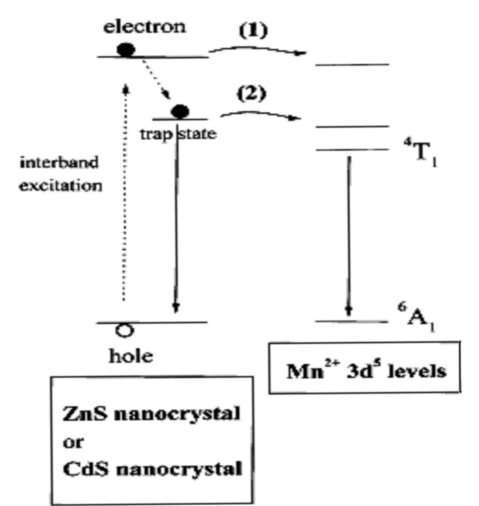

Fig. 1. Schematic diagram of the excitation of $\mathrm{Mn}^{2+}$ in CdS nanocrystals [10].
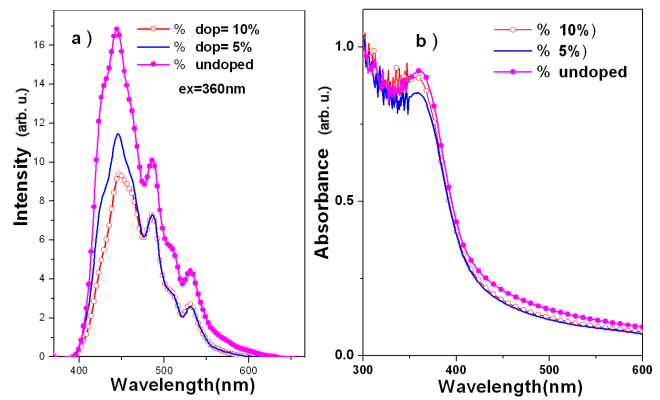

Fig. 2. Luminescence (a) and absorption (b) spectra of CdS:Mn NCs of Table I.

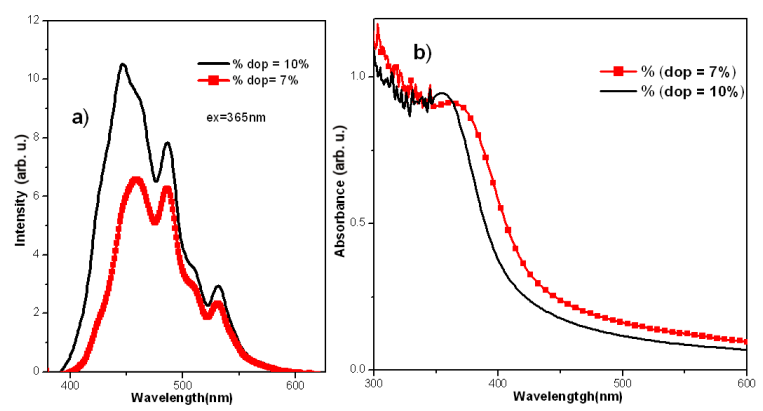

Fig. 3. Luminescence (a) and absorption (b) spectra of CdS:Mn NCs samples in Table II.
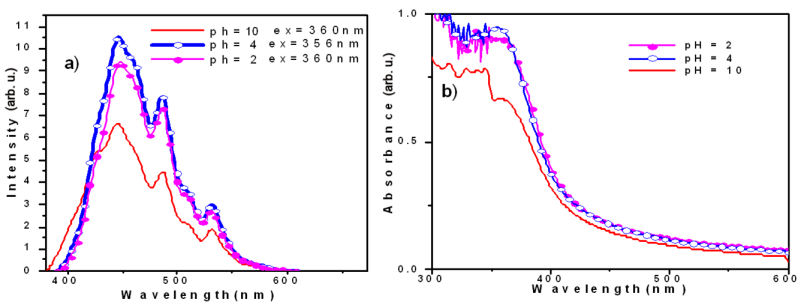

Fig. 4. Luminescence and absorption spectra of CdS:Mn NCs samples of Table III.

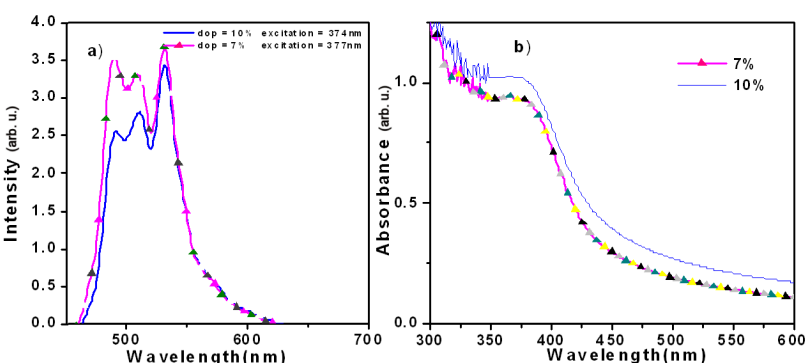

Fig. 5. Luminescence (a) and absorption (b) spectra of CdS:Mn NCs of Table IV.

We explained the synthesis properties of CdS:Mn samples in Tables I-V. In contrast the trap states are located within the semiconductor band gap and hence their emission is usually red-shifted relative to the band edge emission. In addition, the trap state luminescence is often characterized by a large band width reflecting a broad energy distribution of the emitting states. Nonradiative decay pathways associated with unpassivated surface atoms competed with radiative exciton recombination. The characteristic luminescence can consist of relatively sharp emission bands (spectral width typically a few $\mathrm{nm}$ ), but also of broad bands, whose width can exceed $50 \mathrm{~nm}$ in the visible part of the spectrum. Sharp emission bands are characteristic of optical transitions between electronic states with chemical bonding character (almost) the same for ground and excited states that hardly participate in the chemical bonding. These activities in the host material define the luminescence efficiency and can produce a narrowing or broadening of the bandwidth of the spectra [9]. As shown in all figures, we have three peaks that can correspond to band edge, shallow traps, and deep traps.

TABLE I

Synthesis parameters of nanocrystal samples in Fig. 2.

\begin{tabular}{c|c|c|c}
\hline \hline Sample's name & Sample $A$ & Sample $B$ & Sample $C$ \\
\hline Cd and S concentration & $0.01 \mathrm{M}$ & $0.01 \mathrm{M}$ & $0.01 \mathrm{M}$ \\
TG concentration & $0.02 \mathrm{M}$ & $0.02 \mathrm{M}$ & $0.02 \mathrm{M}$ \\
temperature & $\mathrm{RT}$ & $\mathrm{RT}$ & $\mathrm{RT}$ \\
pH value & 2 & 2 & 2 \\
dop. concentration $(\mathrm{Mn})$ & undoped & $5 \%$ & $10 \%$
\end{tabular}

TABLE II

Synthesis parameters of nanocrystal samples in Fig. 3.

\begin{tabular}{c|c|c}
\hline \hline Sample's name & Sample $D$ & Sample $E$ \\
\hline Cd and S concentration & $0.01 \mathrm{M}$ & $0.01 \mathrm{M}$ \\
TG concentration & $0.02 \mathrm{M}$ & $0.02 \mathrm{M}$ \\
temperature & $\mathrm{RT}$ & $\mathrm{RT}$ \\
pH value & 4 & 4 \\
dop. concentration $(\mathrm{Mn})$ & $7 \%$ & $10 \%$
\end{tabular}

Figure 2 shows the PL spectra and absorption spectra of a series of samples with different doping concentration. The PL spectra (Fig. 2a) of the nanocrystal 
TABLE III

Synthesis parameters of nanocrystal samples in Fig. 4.

\begin{tabular}{c|c|c|c}
\hline \hline Sample's name & Sample $F$ & Sample $G$ & Sample $H$ \\
\hline Cd and S concentration & $0.01 \mathrm{M}$ & $0.01 \mathrm{M}$ & $0.01 \mathrm{M}$ \\
TG concentration & $0.02 \mathrm{M}$ & $0.02 \mathrm{M}$ & $0.02 \mathrm{M}$ \\
temperature & $\mathrm{RT}$ & $\mathrm{RT}$ & $\mathrm{RT}$ \\
pH value & 2 & 4 & 10 \\
dop. concentration $(\mathrm{Mn})$ & $10 \%$ & $10 \%$ & $10 \%$
\end{tabular}

TABLE IV

Synthesis parameters of nanocrystal samples in Fig. 6.

\begin{tabular}{c|c|c}
\hline \hline Sample's name & Sample $I$ & Sample $J$ \\
\hline Cd and S concentration & $0.1 \mathrm{M}$ & $0.1 \mathrm{M}$ \\
TG concentration & $0.2 \mathrm{M}$ & $0.2 \mathrm{M}$ \\
temperature & $\mathrm{RT}$ & $\mathrm{RT}$ \\
pH value & 6 & 6 \\
dop. concentration $(\mathrm{Mn})$ & $7 \%$ & $10 \%$
\end{tabular}

samples show three peaks, at 444, 478, and $530 \mathrm{~nm}$, which are attributed to the band edge, shallow and deep traps, respectively. We see that undoped NCs have a narrower size distribution than the $5 \%$ and $10 \%$ doping NCs. Therefore they have the highest emission intensity. But we expected doping $\mathrm{Mn}$ ions to produce better luminescence spectra. The impurity concentrations generally are low in view of the fact that at higher concentration the efficiency of the luminescence process usually decreases (concentration quenching) [11, 15]. It may be that, at high concentration, there is the broad size distribution of NCs and broad emission wavelength, and as a result, low luminescence intensity. Other samples like those in Fig. 3 confirm these results.

As we show in Fig. 4, the luminescence intensity decreases with increasing $\mathrm{pH}$. The absorption spectra in $\mathrm{pH} 4$ have sharper excitonic peaks than $\mathrm{pH} 2$ and 10, respectively. Moreover, it was found that $\mathrm{MnS}$ can create an acidic synthesis solution which produces radiative recombination centers in the CdS host material.

We prepared CdS:Mn nanocrystals at a high precursor concentration with $0.1 \mathrm{M}$. Figure 5 shows that $7 \%$ doping has higher luminescence intensity than the sample with lower precursor concentration and the same doping level (Fig. 3).

Atomic absorption spectroscopy (AAS) analysis demonstrates all $\mathrm{Mn}^{2+}$ ions expended in high precursor concentration (samples $I$ and $J$ ) up to $98 \%$ comparing

Synthesis parameters of nanocrystal samples

TABLE V

in Figs. 7 and 8.

\begin{tabular}{c|c|c}
\hline \hline Sample's name & Sample $K$ & Sample $L$ \\
\hline Cd and S concentration & $0.02 \mathrm{M}, 0.01 \mathrm{M}$ & $0.02 \mathrm{M}, 0.01 \mathrm{M}$ \\
TG concentration & $0.25 \mathrm{M}$ & $0.25 \mathrm{M}$ \\
temperature & $\mathrm{RT}$ & $\mathrm{RT}$ \\
pH value & 2 & 2 \\
dop. concentration $(\mathrm{Mn})$ & $1.18 \%$ & $5 \%$
\end{tabular}

to low concentration samples $(D$ and $E$ ). It means that doping of nanocrystals occurs completely in high precursor concentration. Figure 6 shows the energy dispersive X-ray (EDX) spectroscopy image of CdS:Mn NCs for $76^{\circ} \mathrm{C}$. Figure 6 confirmed that we prepared CdS nanoparticles doped with $\mathrm{Mn}$ ions. Because we used $\mathrm{Al}$ substrate and $\mathrm{Au}$ sputtering, these elements present on EDX pattern.

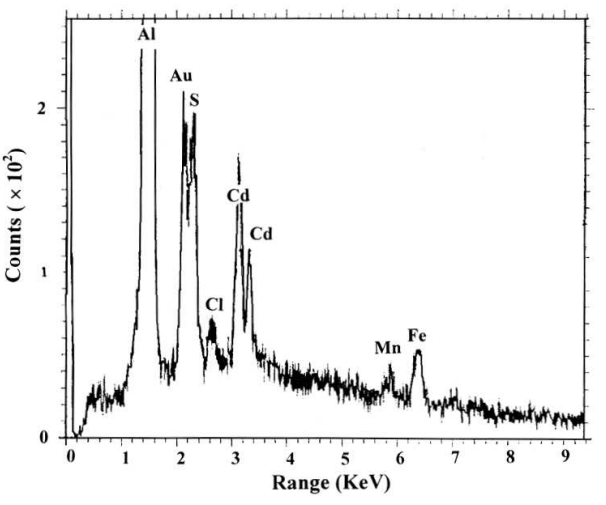

Fig. 6. EDX spectra of Mn doped CdS sample prepared at $76^{\circ} \mathrm{C}$.

As a result, the existence of $\mathrm{Mn}$ ions in NCs deforms the crystal lattice and therefore, disarranges their size distribution.

Also, we investigated the optimize conditions for the best luminescence with different excitation wavelengths and doping concentrations. The samples $K$ and $L$ were prepared according to the conditions mentioned in Table V. As shown in Fig. 7, there is a narrowing of the excitonic absorption peak at $350 \mathrm{~nm}$. We excited sample $K$ with three excitation wavelengths. There is the best luminescence for $360 \mathrm{~nm}$ excitation which is near to band edge wavelength. Two other excited emissions related to the sizes of other nanoparticles were distributed in this sample. We find a red-shift of the band edge emission by varying the excited wavelength [12]. But we have a broad peak at $465 \mathrm{~nm}$ in all of them that is related to the incorporation of nonradiative and radiative recombination.

As can be seen in Figs. 7 and 8, there is a better luminescence intensity for $360 \mathrm{~nm}$ excitation with narrow size distribution compared to others. Also, with increasing $\mathrm{Mn}$ doping concentration from sample $K$ to sample $L$ the luminescence intensity increased.

As shown in Fig. 7, there is better absorption intensity (ABS) comparing to Fig. 8. Therefore we expect it has better luminescence intensity. But when the $\mathrm{Mn}^{2+}$ concentration is increased, more radiative recombination occurs and, as a result, the luminescence intensity increased from sample $K$ to $L$. 


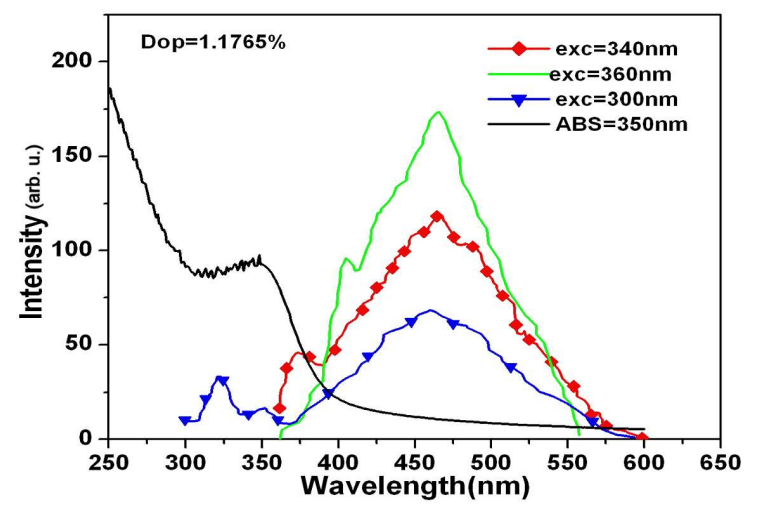

Fig. 7. Luminescence spectra of CdS:Mn NCs of sample $K$ in Table V, under excitation of $300 \mathrm{~nm}, 340 \mathrm{~nm}$ and $360 \mathrm{~nm}$ and absorption spectrum (ABS).

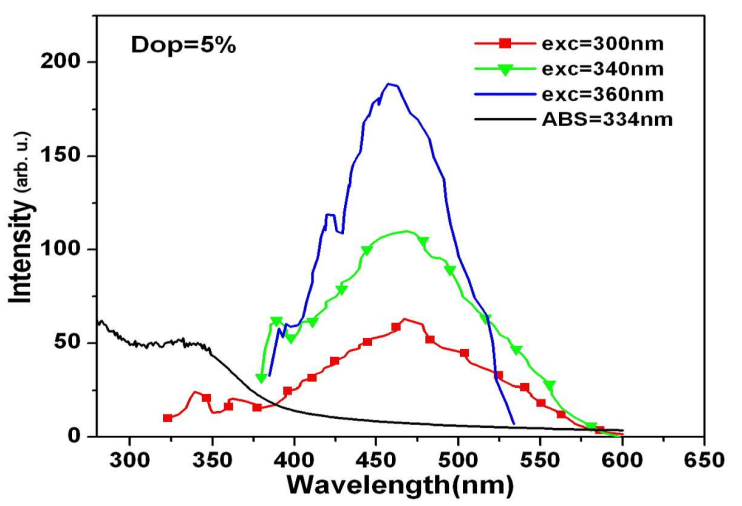

Fig. 8. Luminescence spectra of CdS:Mn NCs of sample $L$ in Table V, under excitation of $300 \mathrm{~nm}, 340 \mathrm{~nm}$ and $360 \mathrm{~nm}$ and absorption spectrum (ABS).

\section{Conclusion}

In conclusion, a simple aqueous solution method has been developed for synthesizing the size-controlled $\mathrm{Mn}^{2+}$ doped CdS nanocrystals with a relatively narrow size distribution. It confirmed that narrow size distribution samples have better luminescence intensity because they have less broadening on wavelength emissions due to their broad sizes. These results indicate that band edge emission is more affective than traps on the luminescence intensity. They do not have noticeable changes in energy by variation of the doping concentrations. It is observed that the luminescence intensity increases with increasing Mn concentration from $1.18 \%$ to $5 \%$. Luminescence intensity in different excitation wavelength shows portion of different size of CdS:Mn nanocrystals on luminescence spectra. We found that by narrowing the size distribution and the amount of doping concentration CdS samples with a high luminescence intensity can be prepared.

\section{Acknowledgments}

The authors are grateful to the University of Guilan for financial support for this research work.

\section{References}

[1] B. Tripathi, F. Singh, D.K. Avasthi, D. Das, Y.K. Vijay, Physica B 400, 70 (2007).

[2] R. Lotfi Orimi, N. Shahtahmasebi, N. Tajabor, Physica E 40, 2894 (2008).

[3] M. Tanaka, Y. Masumoto, Solid State Commun. 120, 7 (2001).

[4] N. Peyghambarian, S.W. Koch, A. Mysyrowicz, Introduction to Semiconductor Optics, Prentice Hall, Englewood Cliffs, NJ 1993.

[5] E. Herz, M.Sc. Thesis, Virginia Polytechnic, Virginia, 2003.

[6] Sh. Liu, F. Liu, H. Guo, Solid State Commun. 115, 615 (2000).

[7] J. Nanda, B.A. Kuruvilla, D.D. Sarma, Phys. Rev. B 59, 7473 (1999).

[8] B. Tripathi, Y.K. Vijay, Solid State Electron. 51, 81 (2007).

[9] Q. Xiao, C. Xiao, L. Ouyang, J. Lumin. 128, 1942 (2008).

[10] M. Tanaka, J. Lumin. 100, 163 (2002).

[11] S. Ronda, Luminescence from Theory to Applications, Wiley-VCH Verlag, Weinheim 2008.

[12] Chu Viet Ha, Vu Thi Hong Hanh, Vu Thi Kim Lien, Pham Thai Cuong, Vu Duc Chinh, Pham Thu Nga, C. Barthou, P. Benalloul, ASIAN J. Sc. Techn. Development 24, 77 (2007).

[13] R. Beaulac, P.I. Archer, D.R. Gamelin, J. Solid State Chem. 181, 1582 (2008).

[14] Sh. Liu, F. Liu, H. Guo, Solid State Commun. 115, 615 (2000).

[15] Ch. Chory, Ch. Remenyi, C. Dem, Phys. Chem. 5, 1639 (2003). 